\title{
Optimum maintenance strategy for deteriorating bridge structures based on lifetime functions
}

\author{
Seung-Ie Yang, Dan M. Frangopol*, Luís C. Neves ${ }^{1}$ \\ Department of Civil, Environmental, and Architectural Engineering, Campus Box 428, University of Colorado, Boulder, CO 80309-0428, USA
}

Received 19 May 2003; received in revised form 28 May 2004; accepted 14 June 2005

\begin{abstract}
The highway networks of most European and North American countries are completed or close to completion. However, many of their bridges are aging, and in the United States alone a very significant part of the about 600,000 existing bridges is considered to be deficient and must be replaced, repaired or upgraded in the short term. The funds available for the maintenance of existing highway bridges are extremely limited when compared with the huge investment necessary, and must, therefore, be spent wisely. In this paper, a model based on lifetime functions for predicting the evolution in time of the reliability of deteriorating bridges under maintenance is presented. This model uses the probability of satisfactory system performance during a specified time interval as a measure of reliability and treats each bridge structure as a system composed of several components. In this manner, it is possible to predict the structural performance of deteriorating structures in a probabilistic framework. In addition, the optimum maintenance strategy is identified using as objective the minimization of the present value of the life-cycle maintenance cost. An existing bridge is analyzed using lifetime functions and its optimum maintenance strategy is found.

(C) 2005 Published by Elsevier Ltd
\end{abstract}

Keywords: Bridges; Maintenance; Lifetime functions; Deteriorating structures; System performance; Optimum maintenance strategy

\section{Introduction} deterioration.
The highway networks of most European and North American countries are completed or close to completion. As a result, highway agencies face a decrease in the need for new structures and, on the other hand, a very significant increase in the number of bridges that need to be repaired or replaced in the short term. In the United States a very significant part of the existing bridges is considered to be deficient and must be repaired, upgraded or replaced in the near future. As a result, in the last decade, research has shifted from the design of new bridges to the assessment of existing bridges and prediction of their performance

\footnotetext{
* Corresponding author. Tel.: +1 303492 7165; fax: +1 3034927317

E-mail address: dan.frangopol@colorado.edu (D.M. Frangopol).

1 Visiting Researcher, Department of Civil, Environmental, and Architectural Engineering, University of Colorado, Boulder, CO 803090428, USA. On leave from: Department of Civil Engineering, University of Minho, Guimarães, Portugal.
}

Due to the limited funds available for upgrading and maintaining the performance of existing bridges at acceptable levels, highway agencies, governments and researchers have tried to develop models that predict optimum strategies to be used in the maintenance planning for existing bridges, keeping them safe and serviceable by using the smallest possible investment.

The current bridge management systems use visual inspection results to assess bridge safety $[12,16,21]$. These systems are based on component level analysis, disregarding overall system effects such as redundancy, ductility, and component reliability importance. It has long been recognized that several reliability measures (e.g., reliability index and probability of survival) are consistent and invariant indicators of structural safety. The reliability index of a structure can be higher or lower than that of its critical component, for parallel and series systems, respectively. Therefore, the evaluation of the overall structural system safety is of paramount importance in assessing the safety of new and existing bridges. 
The deterioration of a bridge depends on several parameters (e.g., environmental conditions, traffic volume, and quality of workmanship) that cannot be accurately predicted. Consequently, bridge deterioration must be modeled in a probabilistic manner, using random variables for the parameters defining the deterioration process. To keep the reliability of a bridge above a minimum target level during a specified period of time, maintenance actions must usually be applied. In general, these actions reduce the rate of increase of the cumulative time system failure probability [5]. Several maintenance strategies satisfying the above requirements are possible. In general, the cost of each feasible maintenance strategy is different from the others. The optimum maintenance strategy, associated with minimum present value of cumulative cost, must be found.

Most decisions in bridge maintenance must to be made with a binary type of information based on visual inspections where defects are found or not found. To be able to correctly assess and predict the performance of existing structures using only this information, the performance must be indicated using the probability of occurrence of a defect rather than a continuous damage model. This approach is less accurate than the continuous damage model approach, but can be implemented using the information currently available on most structures.

In this paper, a model based on lifetime functions for predicting the evolution in time of the reliability of deteriorating bridges under maintenance is presented. This model uses the probability of satisfactory system performance during a specified time interval as a measure of reliability and treats each bridge structure as a system composed of several components. In this manner, it is possible to predict the structural performance of deteriorating structures in a probabilistic framework. In addition, the optimum maintenance strategy is identified using as objective the minimization of the present value of the life-cycle maintenance cost. An existing bridge is analyzed using lifetime functions and its optimum maintenance strategy is found. Probabilistic approaches to deteriorating and/or maintenance of existing structures can also be found in $[3,20,4,8]$.

\section{System reliability and reliability importance based on lifetime functions}

defined as follows:

$\phi(\mathbf{x})= \begin{cases}0 & \text { if the system has failed } \\ 1 & \text { if the system is functioning. }\end{cases}$

where $\mathbf{x}=$ vector containing the state of each component.

Structures modeled as series and parallel systems are safe when all and at least one of their components are safe, respectively. For these systems, the associated structure functions are, respectively, defined as:

$$
\begin{aligned}
& \phi(\mathbf{x})=\min \left(x_{1}, x_{2}, \ldots, x_{n}\right)=\prod_{i=1}^{n} x_{i} \\
& \phi(\mathbf{x})=\max \left(x_{1}, x_{2}, \ldots, x_{n}\right)=1-\prod_{i=1}^{n}\left(1-x_{i}\right) .
\end{aligned}
$$

A coherent system [17] is a system that will not upgrade if a component degrades (i.e., $\phi(\mathbf{x})$ is non-decreasing in $\mathbf{x}$ ). For a given structure, modeled as a coherent system, the associated structure function can be obtained by modeling the system as series of parallel components. This system can be successively reduced by using Eqs. (3) and (4) to a single equivalent component whose structure function is defined in terms of all components. However, the state of each component can only be expressed in probabilistic terms by considering components defined by their probabilities of survival.

So far, components and system performance have only been considered at a particular point in time. However, due to material deterioration and/or increase in environmental and/or mechanical loadings the reliability of a structure or component under no maintenance is a non-increasing function of time, called the survivor function $S(t)$. This is a particular type of lifetime distribution function that includes also the hazard function and the mean residual life function, among others. In this study, two survivor functions are considered: Weibull and exponential power. These non-increasing functions are 1 and 0 at $t=0$ and $t \rightarrow \infty$, respectively. Figs. 1 and 2 show the effects of the number of independent components, each characterized by the same survivor function (i.e., exponential power function with a failure rate $\lambda$ of $0.005 /$ year), on the survivor function of a series and a parallel system up to 10 components, respectively, considering a lifetime of 75 years.

The survivor functions of a series-parallel system of four components with different exponential power survivor functions ( $\lambda=0.005 /$ year for components 2,3 , and, 4 and $\lambda$ varying from 0.001 /year to 0.01 /year for component 1 ), analyzed over a lifetime period of 75 years, are shown in Fig. 3. As expected, a change in the survivor function of component 1 leads to a significant change in the system survivor function. Additional examples on the effects of the parameters of exponential and Weibull survivor functions are provided in $[25,26]$.

In general, the components of a structural system have different impacts on the overall system reliability. According to Leemis [17], "the component with the largest reliability 


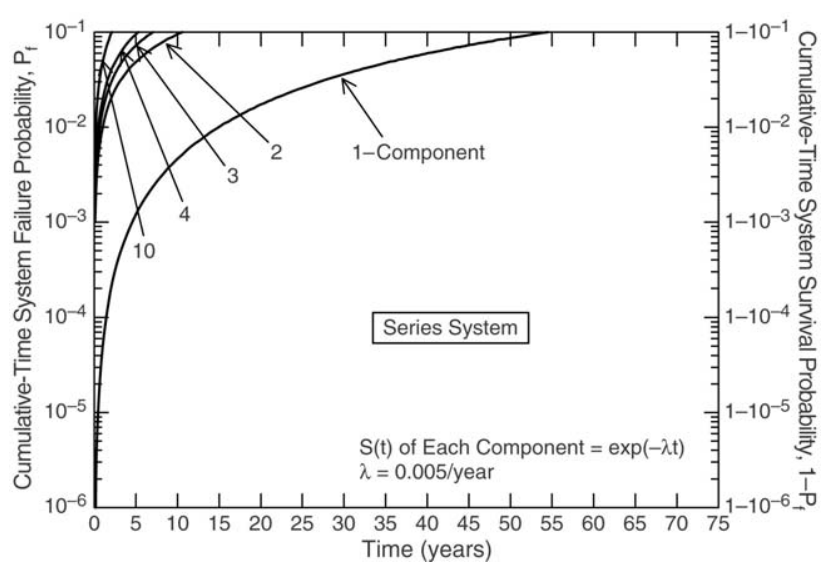

Fig. 1. Effect of number of components on cumulative-time failure probability of series systems.

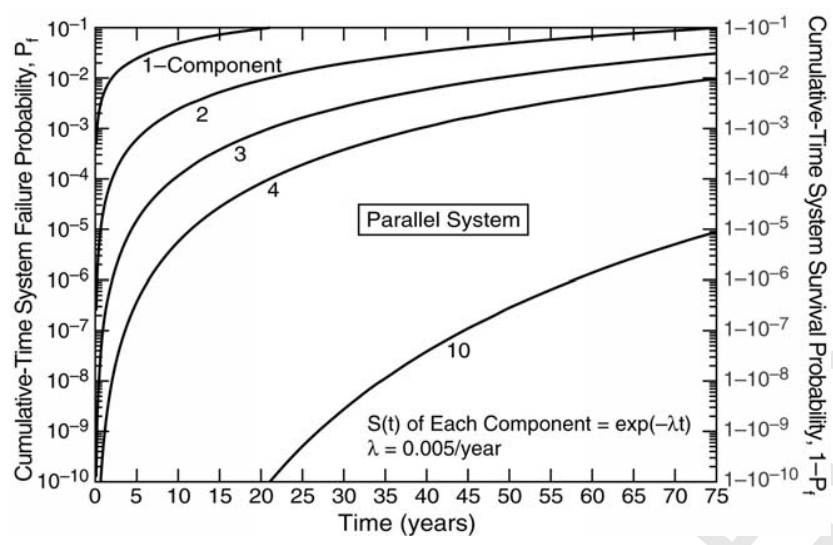

Fig. 2. Effect of number of components on cumulative-time failure probability of parallel systems.

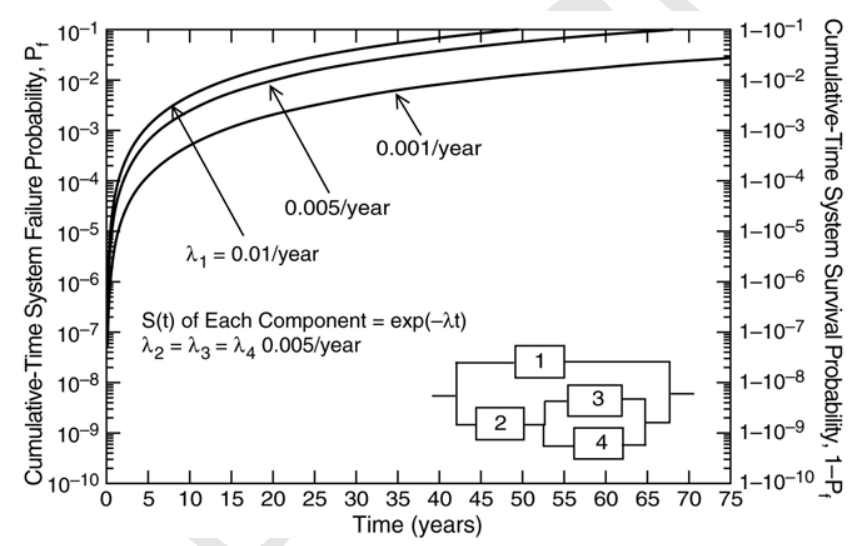

Fig. 3. Effect of failure rate of component 1 on cumulative-time failure probability of a four-component system.

1 importance is that component for which an increase in 2 its reliability corresponds to the largest increase in the 3 system reliability". Consequently, the reliability importance 4 of component $i, I_{r}(i)$, is as follows [17]:

${ }_{5} \quad I_{r}(i)=\frac{\partial r(\boldsymbol{p})}{\partial p_{i}}$

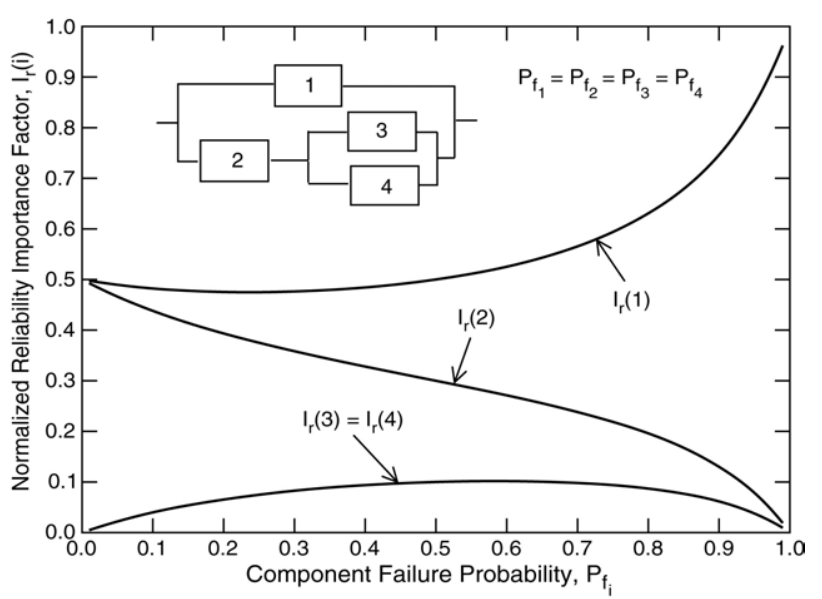

Fig. 4. Normalized reliability importance factors for a four-component system versus component failure probability.

where $r(\boldsymbol{p})=$ system reliability and $p_{i}=$ probability of failure of component $i$.

This factor can be normalized as follows [11]:

$I_{r}^{0}(i)=\frac{I_{r}(i)}{\sum_{i=1}^{n} I_{r}(i)}$

where $I_{r}^{0}(i)=$ normalized reliability importance factor of component $i$, varying from 0 (not relevant to system reliability) to 1 (only relevant component to system reliability), and $n=$ number of components. Since the system reliability is time dependent so are the reliability importance factors $I_{r}(i)$ and $I_{r}^{0}(i)$.

In Fig. 4 the normalized reliability importance factor $I_{r}^{0}(i)$ of each of the four components of the series-parallel system analyzed in Fig. 3 is shown for different probabilities of failure of the iso-reliability components. As expected, component 1 , due to its critical function in the system, has the highest reliability importance factor over all the range of component failure probabilities considered.

In most cases, the failure rate of a component is not known a priori and, as a result, it must be treated as a random variable. To illustrate the effect of randomness of the failure rate on the survivor function of a system, Fig. 5 shows the evolution in time of the probability of survival of the fourcomponent system defined in Fig. 3 considering the same random failure rates for all components defined by a uniform distribution varying from 0.00413 /year to 0.00586 /year. As shown, the range of possible values of the system survival probability depends on the randomness of the failure rate of components.

\section{Preventive and essential maintenance models}

As previously indicated, the reliability of a structure can be kept above a specified threshold by applying maintenance actions. These actions can be divided in two major groups: (i) preventive actions; and (ii) essential actions. Preventive 


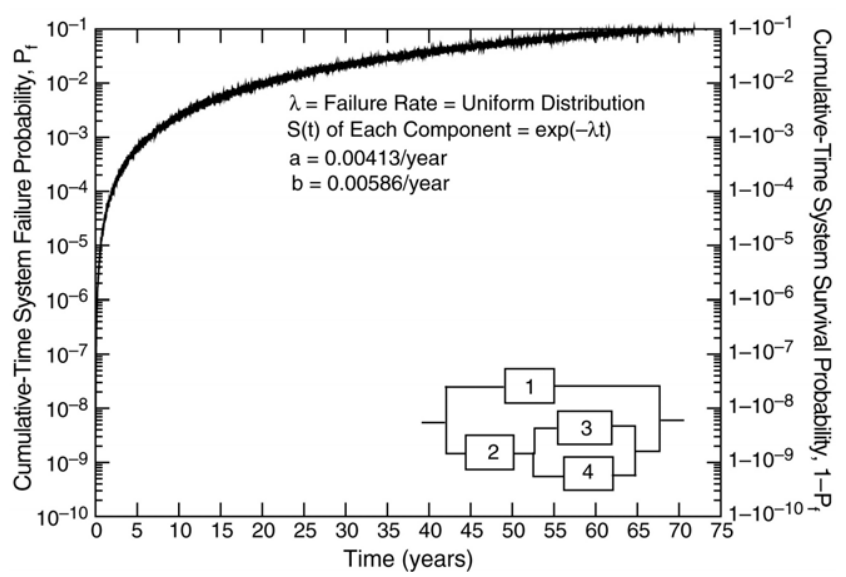

Fig. 5. Cumulative-time failure probability of a four-component series-parallel system considering random failure rates of components.

33 where $t_{0(i-1)}=$ time of damage initiation considering $i-1$

\begin{abstract}
maintenance actions (such as painting, silane treatment, and cathodic protection) are defined as scheduled maintenance actions applied to functioning components. The justification for preventive maintenance action is that if not undertaken it will require more funds at a later stage to keep the component from becoming critical [2,9]. Preventive actions applied to non-deteriorated components are designated as proactive and their objective is to delay the time of damage initiation [13]. Preventive maintenance actions applied to deteriorated components are denoted as reactive, and they aim at eliminating or reducing the effects of the deterioration process. Several maintenance models in a probabilistic context were developed by Frangopol et al. [10], Bris et al. [1], Kobbacy and Jeon [14], and Lam and Zhang [15], among others. In this section, both preventive and essential maintenance models are briefly summarized. Additional information is provided in [24] and [26].
\end{abstract}

\subsection{Proactive preventive maintenance}

Due to the lack of data on proactive maintenance models, expert judgment is generally used to define the effect of applying this type of maintenance. In this study, it is assumed that each proactive maintenance action (applied before damage initiation) postpones the initial time of damage initiation under no maintenance, $t_{0}$, to [26]:

$t_{0 i}=t_{0}+i \cdot \frac{t_{p i}}{2}$

where $t_{0 i}=$ time of damage initiation considering $i$ proactive maintenance actions, and $t_{p i}=$ time interval between maintenance actions. In order to compute the number $i$ of proactive maintenance actions necessary to obtain a specified value of $t_{0 i}$, the following constraint must be satisfied: proactive maintenance actions.

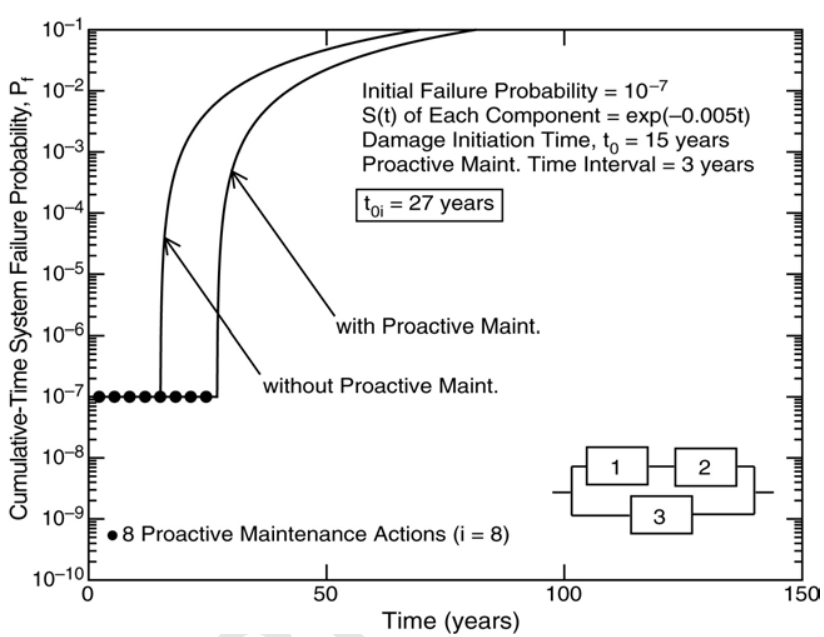

Fig. 6. Effect of proactive maintenance on a three-component series-parallel system.

An example of the effect of proactive maintenance on cumulative-time system failure probability is shown in Fig. 6, considering a three-component series-parallel system with a probability of survival of each component described by an exponential function. Both proactive maintenance and no maintenance strategies are considered. In this example, the damage initiation time of both components and system is extended from $t_{0}=15$ years (no maintenance) to $t_{0 i}=27$ years (under eight preventive maintenance actions applied every three years, $t_{p i}=3$ years, to all components).

\subsection{Reactive preventive maintenance}

In this study, the reactive maintenance model proposed by Kececioglu [13] is used. This model considers that, if reactive maintenance is applied at regular time intervals, $t_{p}$, the survivor function is as follows [13,26]:

$S_{t_{p}}(t)=\left[S_{t}\left(t_{p}\right)\right]^{j} S_{t}(\tau)$

where $S_{t}=$ survivor function under no maintenance, $S_{t_{p}}(t)=$ survivor function under reactive preventive maintenance at time $t, t_{p}=$ time interval between applications of reactive preventive maintenance, $j=$ number of applications of reactive preventive maintenances before time $t$, and $\tau=$ time since last application.

An example of the effect of reactive preventive maintenance is presented in Fig. 7. In this figure each component of the deteriorating two-component parallel system is subjected to reactive maintenance at different time intervals, $t_{p}$. The survivor function of each independent component is $\exp (-0.01 t)$. As shown in this figure, the effect of each reactive preventive maintenance action is to reduce the slope of the cumulative survival function to its initial value (at $t=0$ ). As expected, more frequent applications lead to higher probabilities of system survival.

If reactive preventive maintenance is applied only to some components of a system (e.g., two out of four 


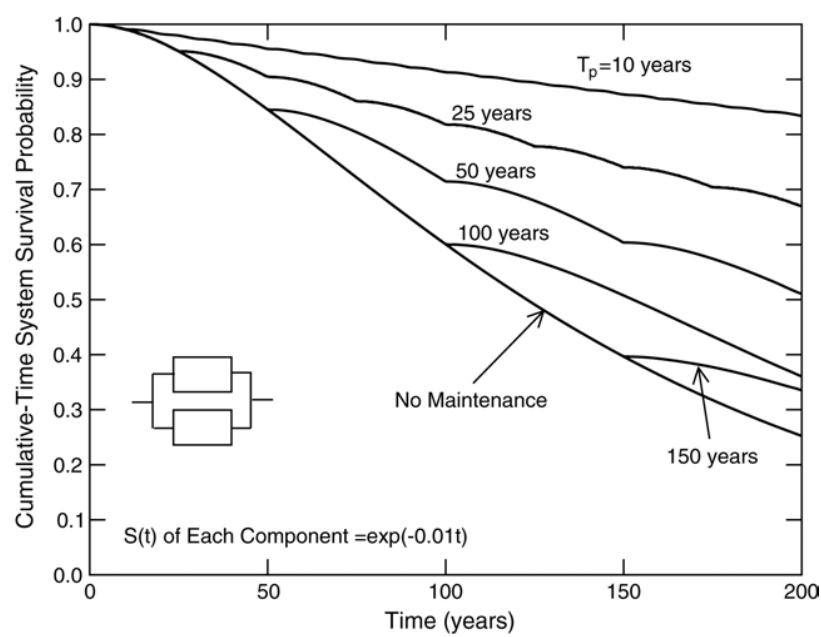

Fig. 7. Cumulative-time system survivor probability of a two-component parallel system under reactive maintenance applied to both components at different time intervals.

girders), Eq. (9) is no longer valid and reliability importance 2 factors must be taken under consideration as indicated by Yang [24]. As an example, Fig. 8 shows the results obtained considering that one, several, or all the three deteriorating components, characterized by the survivor function $S_{t}=$ $\exp (-0.005 t)$, of a series-parallel system are under cyclic reactive preventive maintenance at five years' interval. Component 3, being the most important, has the largest effect on the cumulative-time system failure probability.

\subsection{Essential maintenance}

Essential maintenance actions are applied to failed or close to failure components. Since it is desirable to repair or replace such components as soon as possible, such maintenance actions cannot be scheduled a priori. In this work the only essential maintenance action considered is replacement of one, several, or all components of a system, resulting in the restoration of the condition of such components to their initial values (at $t=0$ ).

The three-component system shown in Fig. 9 is used to explain the essential maintenance model. Each component has an exponential survivor function. It is assumed that all three components are independent and their failure rate is $0.0005 /$ year. The survivor function under no maintenance of the three-component system in Fig. 9 is indicated in [25]. If essential maintenance is performed on one, several, or all components, the survivor function of the system depends on the time since maintenance was last applied to component $i(i=1,2,3)$. The three essential maintenance actions considered in Fig. 9 are replacement of component 1 , component 2, and all three components at 10, 20, and 40 years, respectively. As indicated in Fig. 9, replacement of components 1 or 2 causes a relatively small reduction in the system failure probability.

Based on an extension of the essential maintenance model presented in this section, using survivor functions for

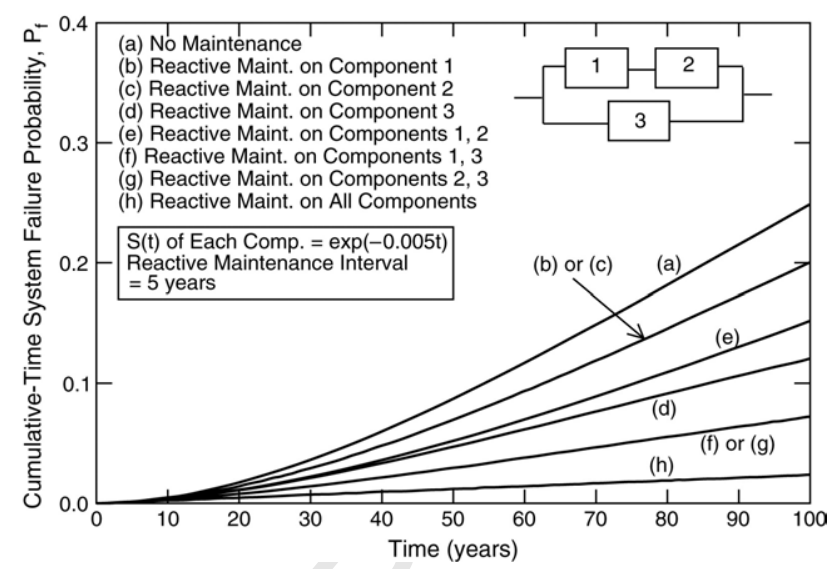

Fig. 8. Cumulative-time system failure probability of a three-component series-parallel system under reactive maintenance applied to one, several, or all components.

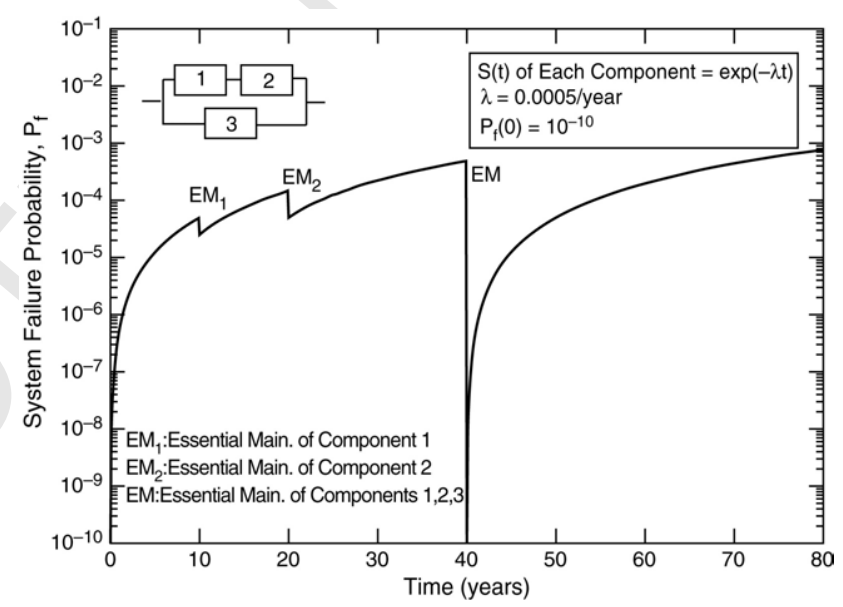

Fig. 9. System failure probability of a three-component series-parallel system under essential maintenance

each component of a series-parallel system, an optimum maintenance strategy is formulated next and applied to an existing bridge.

\section{Optimization and data on lifetime functions}

The methodology used for optimizing the essential maintenance strategies is adapted from that proposed by Estes and Frangopol [7]. It consists of the following nine steps:

(a) Construct a system model of the overall structure as a series-parallel combination of individual components and establish a time horizon for the system;

(b) Define the survivor function to be used for each component;

(c) Compute the survivor function under no maintenance for the system model considered in step (a);

(d) Establish a system reliability threshold, at which maintenance must be applied; 
(a)

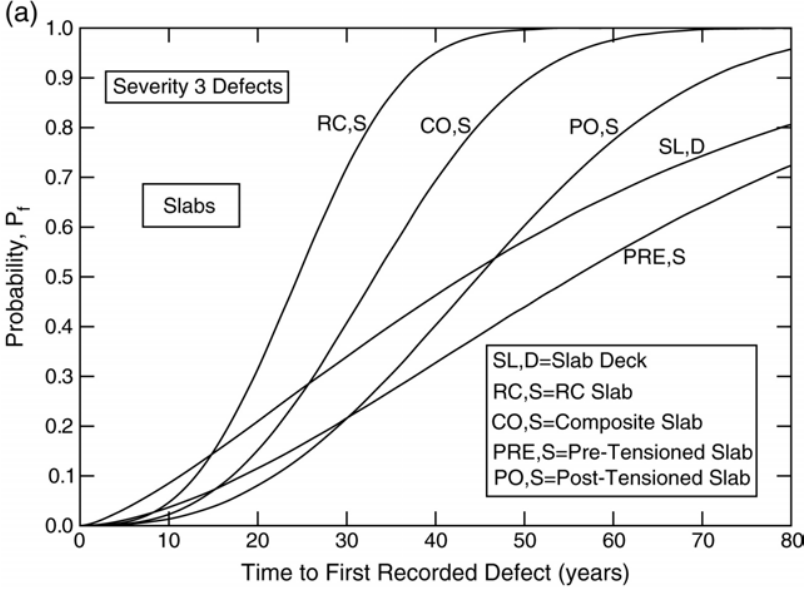

(b)

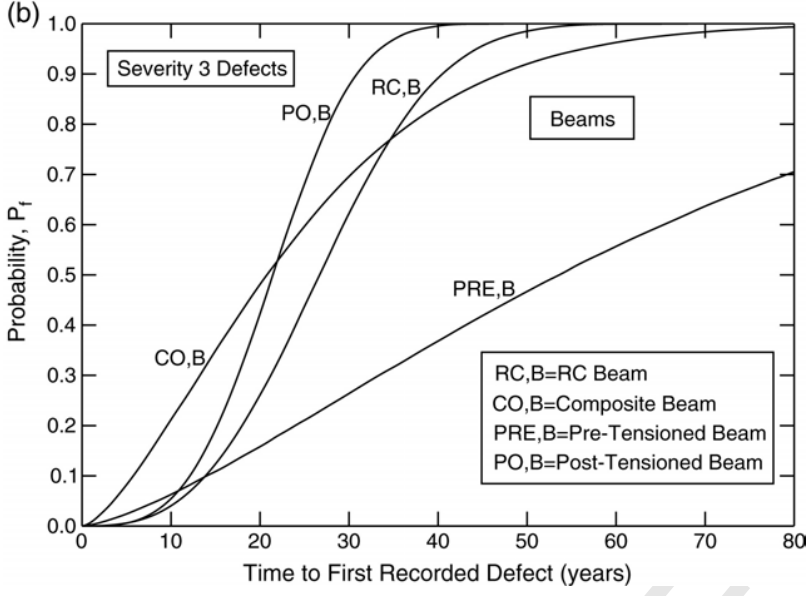

Fig. 10. Cumulative probability of occurrence of severity 3 defect in: (a) slabs, and (b) beams.

(e) Determine all possible maintenance actions and their associated costs;

(f) Determine all maintenance strategies (i.e., combination of several maintenance actions during the time horizon);

(g) Compute the system survivor function for each maintenance strategy;

(h) Compute the present values of lifetime cost for each maintenance strategy; and

(i) Determine the optimum solution based on the minimum present value of lifetime cost.

In this study, data compiled by Maunsell [18] for the serviceable life of highway structures and their components is used. The service life is defined as the time taken for a significant defect to be recorded by an inspector. The severity of a defect is classified as follows [18]: Severity 1: no significant defects; Severity 2: minor defects of a nonurgent nature; Severity 3: defects which shall be included for attention within the next annual maintenance program; and Severity 4: the defect is severe and urgent action is needed.

Data on the lifetime functions corresponding to each of these severities is reported in [18] for different components of the most common types of highway bridges. As an example, using the Weibull distribution parameters of
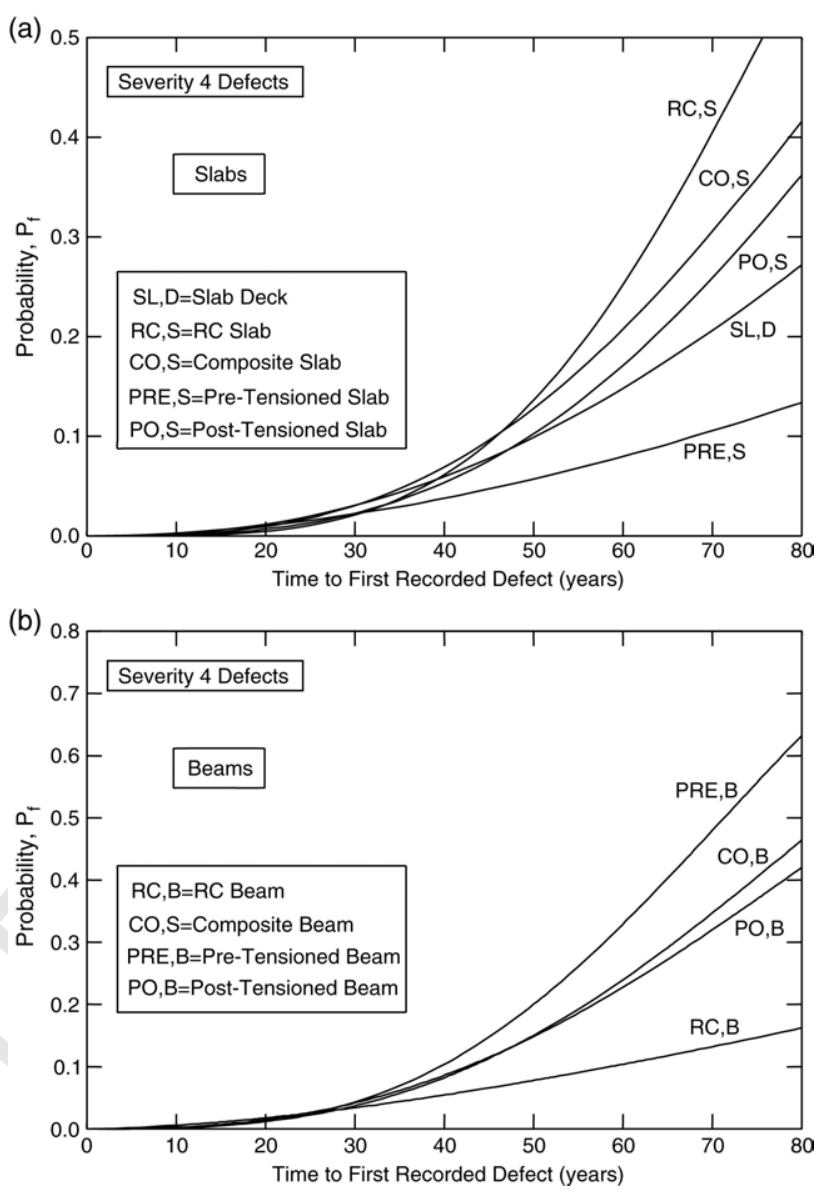

Fig. 11. Cumulative probability of occurrence of severity 4 defect in: (a) slabs, and (b) beams.

service life for severity 3 and 4 defects provided in [18], Figs. 10 and 11 show the cumulative-time probabilities of the first recorded defect for different types of slabs and beams. The Weibull distribution has been shown to properly model aging and to analytically derive the conditional probability density function of the residual lifetime when the current age is provided [23]. As indicated in Figs. 10 and 11 for severity 3 and 4 defects, respectively, there is significant dispersion of the probabilities of occurrence of the same severity defect among different types of elements and materials.

\section{Colorado state highway bridge E-17-AH}

As existing bridge located in Colorado, analyzed previously by a system reliability index approach [7], is presented herein as a case study example using the lifetime function approach. Bridge E-17-AH is located on 40th Avenue (State Highway 33) between Madison and Gardfield Streets in Denver, Colorado. The bridge has three simple spans of equal length $(13.3 \mathrm{~m})$ and a total length of $42.1 \mathrm{~m}$. The deck consists of a $22.9 \mathrm{~cm}$ layer of reinforced concrete and a $7.6 \mathrm{~cm}$ surface layer of asphalt. The east-west bridge has two lanes of traffic in each direction with an average 
daily traffic of 8,500 vehicles. The roadway width is $12.18 \mathrm{~m}$ with $1.51 \mathrm{~m}$ pedestrian sidewalks and handrailing on each side. The bridge offers $6.8 \mathrm{~m}$ of clearance for the railroad spur that runs underneath. There is no skew or curvature. The slab is supported by nine standard-rolled, compact, and non-composite steel girders. The girders are stiffed by end diaphragms and intermediate diaphragms at the third points. Each girder is supported at one end by a fixed bearing and an expansion bearing at the other end. The elevation and cross-section of this nine-girder bridge are indicated in [6], and [25]. A comprehensive description of this bridge can be found in [6].

In this study, failure of a component is defined as occurrence of a defect of severity 4 since this type of defect is relevant enough to justify the application of essential maintenance actions. No distinction is made among different sources of structural defects. As a result, the defects considered include those caused by corrosion, excessive loading, or fatigue, among other sources. Studies considering defects due to various causes including fatigue and corrosion in a probabilistic context can be found in [27,4], and [19].

Weibull functions are adopted to model the probability of defect occurrence as they are the best fit of the data summarized in [18]. The occurrence of the defects in the reinforced concrete slab deck and steel girders of the bridge E-17-AH is modeled by a Weibull distribution with the shape and scale parameters $\kappa$ and $\lambda$ as follows [18]: slab deck $(\kappa=2.37$ and $\lambda=0.0077 /$ year $)$ and girders $(\kappa=2.86$ and $\lambda=0.0106 /$ year).

Due to redundancy in multi-girder bridge types, singlegirder failure does not cause bridge failure. If one girder fails, load redistribution takes place and, usually, the overall bridge is capable of carrying additional loads. Multi-girder bridges can be modeled, in system reliability analysis, as a combination of series and parallel components. For the bridge analyzed, the following failure modes are considered: (i) failure of any external girder or any two adjacent internal girders or deck failure cause the bridge failure; (ii) any two adjacent girder failures or deck failure cause the bridge failure; (iii) any three adjacent girder failures or deck failure cause the bridge failure. These system models, denoted by I, II, and III, respectively, are shown in Fig. 12. In this figure, the failure function $\mathrm{D}$ corresponds to the occurrence of a severity 4 defect in the deck, and the failure functions $\mathrm{G} 1, \mathrm{G} 2, \ldots, \mathrm{G} 9$ correspond to the occurrence of a severity 4 defect in girders G1, G2, ., G9, respectively. Each of the proposed models is associated with a different level of acceptable damage. This level increases from model I to III. Consequently, models III and I are associated with the least and most frequent applications of maintenance, respectively. The choice of the most adequate system model must be, in each situation, made by the bridge owner, considering the available funds, and the importance and redundancy of the structure, among other factors. The maintenance options
Bridge System I

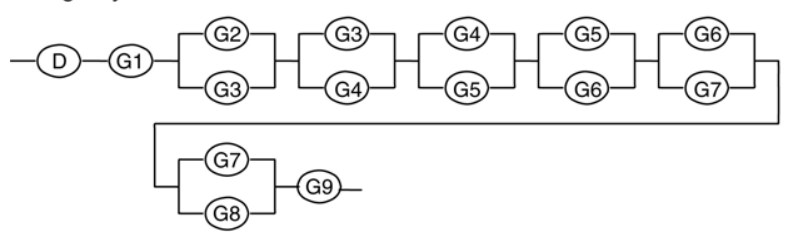

Bridge System II

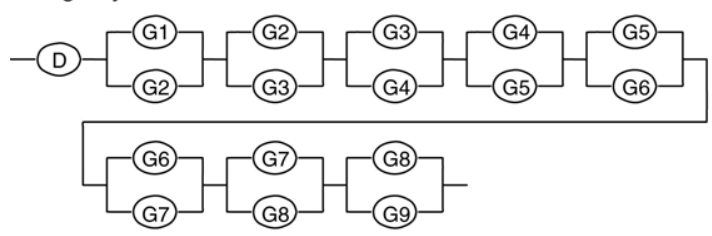

Bridge System III
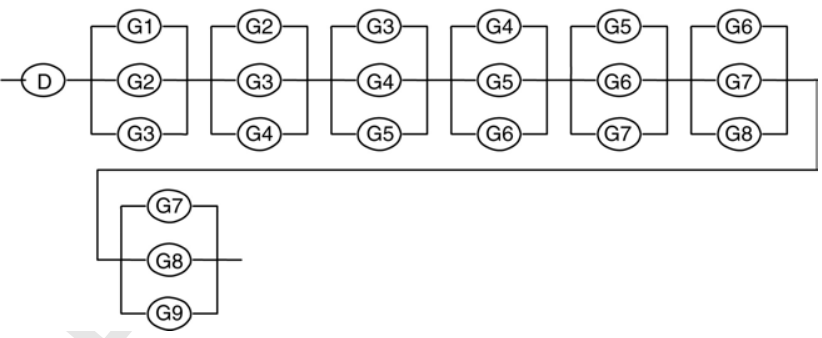

Fig. 12. System models for bridge E-17-AH.

considered for this bridge as well as the associated costs are presented in Table 1 [7].

Table 1

Maintenance actions and their associated costs [7]

\begin{tabular}{lll}
$\begin{array}{l}\text { Maintenance } \\
\text { identification } \\
(1)\end{array}$ & $\begin{array}{l}\text { Maintenance } \\
\text { action } \\
(2)\end{array}$ & $\begin{array}{l}\text { Cost } \\
(1996 \text { US\$) } \\
(3)\end{array}$ \\
\hline 1 & Replace deck & $\$ 225,600$ \\
2 & Replace exterior girders & $\$ 229,200$ \\
3 & Replace deck and exterior girders & $\$ 341,800$ \\
4 & Replace superstructure & $\$ 487,100$ \\
\hline
\end{tabular}

In order to obtain the optimum maintenance strategy, it is necessary to establish the minimum acceptable system probability of occurrence of a defect of severity 4 . In this study, this minimum acceptable system probability level is assumed to be $10^{-2}$ and the target service life is 75 years. All possible combinations of maintenance actions are considered in order to increase the service life to 75 years with the target system probability of $10^{-2}$.

For comparing funds spent at different times the present value of cost

$C_{\mathrm{PV}}=\frac{C}{(1+v)^{t}}$

must be used, where $C_{\mathrm{PV}}=$ present value of maintenance cost, $C=$ cost of maintenance action at time of application, $v=$ discount rate of money, and $t=$ time of application of 
1 maintenance. Historically, discount rates oscillate between $2 \%$ and $8 \%$ [22]. In this study discount rates of $0,2,4,6$, and $8 \%$ are used. The optimization procedure is described next for a discount rate of $2 \%$. However, results are provided for all values of discount rate considered.

For case I (see Fig. 12), system failure is defined as a severity 4 defect being found in the deck, or in an external girder, or in any two adjacent interior girders. As a result, the deck and the exterior girders have a very significant reliability importance. From all systems in Fig. 12 system I is the less redundant and, as a result, the one for which essential maintenance is necessary sooner ( $t=12$ years). In Fig. 13, the four possible maintenance actions $(1,2,3$, and 4 in Table 1) at year 12 are compared in terms of lifetime extension and present value of cost using a discount rate of money of $v=2 \%$. Comparing the present value of cost of each maintenance option per year of increase of service life (i.e., the cost effectiveness) the optimum action at time $t=12$ years is replacement of deck and exterior girders (maintenance action 3). After applying maintenance action 3 at year 12, a second maintenance action must be applied at year 24. At this time the interior girders are more deteriorated than the other components and must be replaced. As a result, at $t=24$ years, maintenance action 4 (replacement of superstructure) is chosen. The replacement of all components leads to a repetition of the lifetime function observed in the first 24 years (Fig. 14). As a result, cyclic maintenance composed of action 3 followed by action 4 is applied until year 72. At this time a less expensive maintenance action (action 2 in Table 1) is suitable to extend the service life beyond the time horizon ( 75 years). The resulting system probability of occurrence of defect 4 associated with the optimum maintenance strategy $3 @ 12$, 4@24,3@36,4@48,3@60, and 2@72(where3@12 means maintenance action 3 applied at year 12) is shown in Fig. 14. The present value of the maintenance cost associated with this strategy, considering $2 \%$ discount rate, is $\$ 1,083,174$ (1996 US\$).

For case II in Fig. 12, system failure is defined as finding a severity 4 defect in the deck or in any two adjacent girders. In this system model no distinction between interior girders and exterior girders is made. This system is more redundant than system I and, as a result, the first maintenance action is applied later and the time interval between maintenance actions is larger (Fig. 15). As indicated in Fig. 16, the threshold system probability of $10^{-2}$ is achieved after 18 years (instead of 12 years for system I). At this time, due to the higher reliability importance of the deck, maintenance option 1 (replacement of the deck) is optimum. At year 28 a second essential maintenance action must be applied. As for case I, the girders are now more deteriorated and must be replaced. Since there is no distinction between interior and exterior girders for the reliability of this system, maintenance option 4 (replace superstructure) is optimum at this time. As all components are repaired (Fig. 16), a repetition of the lifetime function observed in the first 28

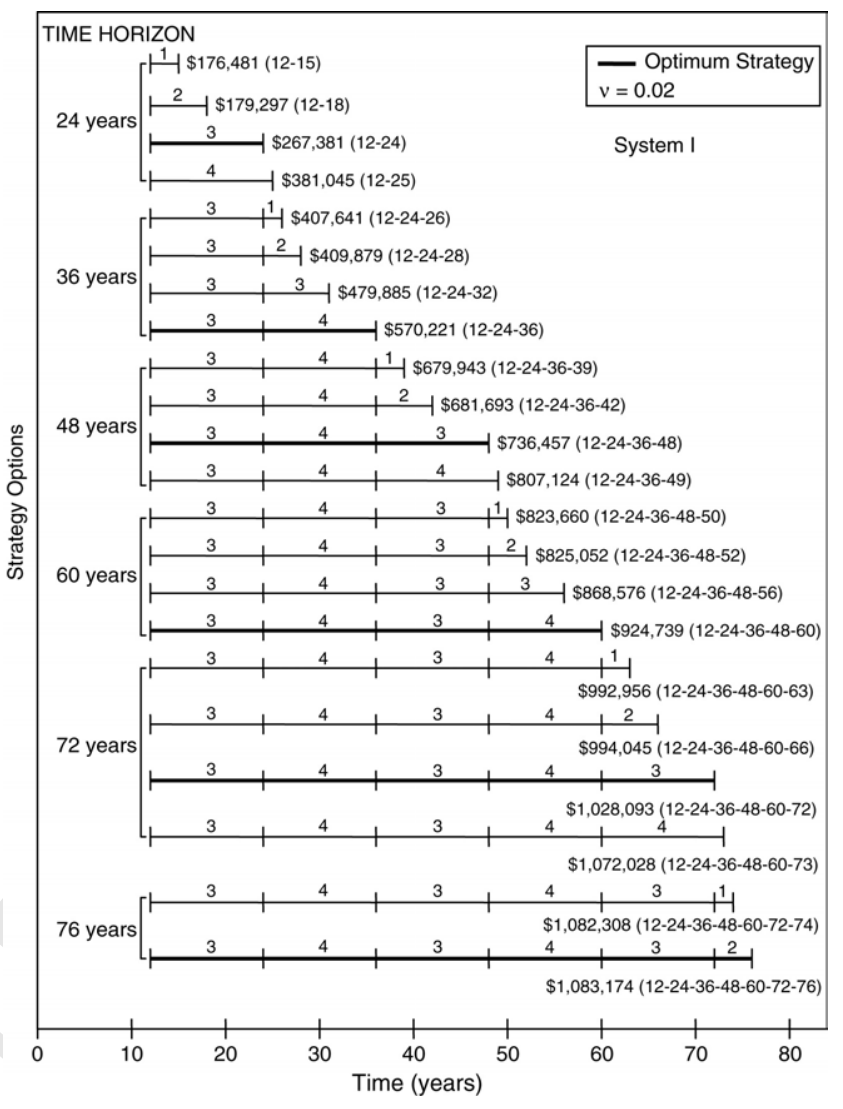

Fig. 13. Optimization of maintenance strategy for bridge system I.

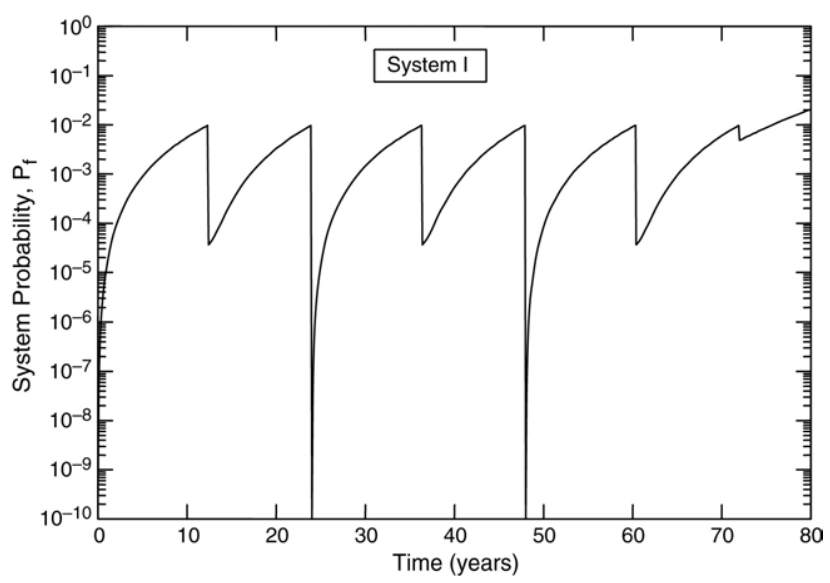

Fig. 14. System probability of occurrence of severity defect 4 under optimum maintenance strategy for bridge system I.

years occurs. As a result, a cycle composed by maintenance action 1 followed by action 4 is repeated until the service life is greater or equal to the time horizon of 75 years (see Fig. 15).

Finally, for case III, system failure is defined as finding a severity 4 defect in the deck or in any three adjacent girders. For this system model, analyzed in [25], the results are presented in Figs. 17 and 18.

In Table 2, the present values of optimum lifetime cost of the three system models in Fig. 12 are presented considering 
Table 2

Comparison of optimum costs for different bridge system models and discount rates

\begin{tabular}{|c|c|c|c|c|c|}
\hline \multirow{2}{*}{$\begin{array}{l}\text { Bridge system model } \\
\text { (1) }\end{array}$} & \multicolumn{5}{|c|}{ Optimum lifetime maintenance cost (1996 US\$) } \\
\hline & $\begin{array}{l}v=0 \% \\
(2)\end{array}$ & $\begin{array}{l}v=2 \% \\
(3)\end{array}$ & $\begin{array}{l}v=4 \% \\
(4)\end{array}$ & $\begin{array}{l}v=6 \% \\
(5)\end{array}$ & $\begin{array}{l}v=8 \% \\
\text { (6) }\end{array}$ \\
\hline I & $2,228,800$ & $1,083,174^{\mathrm{a}}$ & 601,910 & 370,528 & 245,476 \\
\hline II & $1,651,000$ & $739,098^{b}$ & 375,560 & 209,997 & 125,682 \\
\hline III & $1,163,900$ & $526,453^{c}$ & 268,039 & 149,320 & 88,949 \\
\hline
\end{tabular}

${ }^{a}$ See Figs. 13 and 14.

b See Figs. 15 and 16.

c See Figs. 17 and 18.

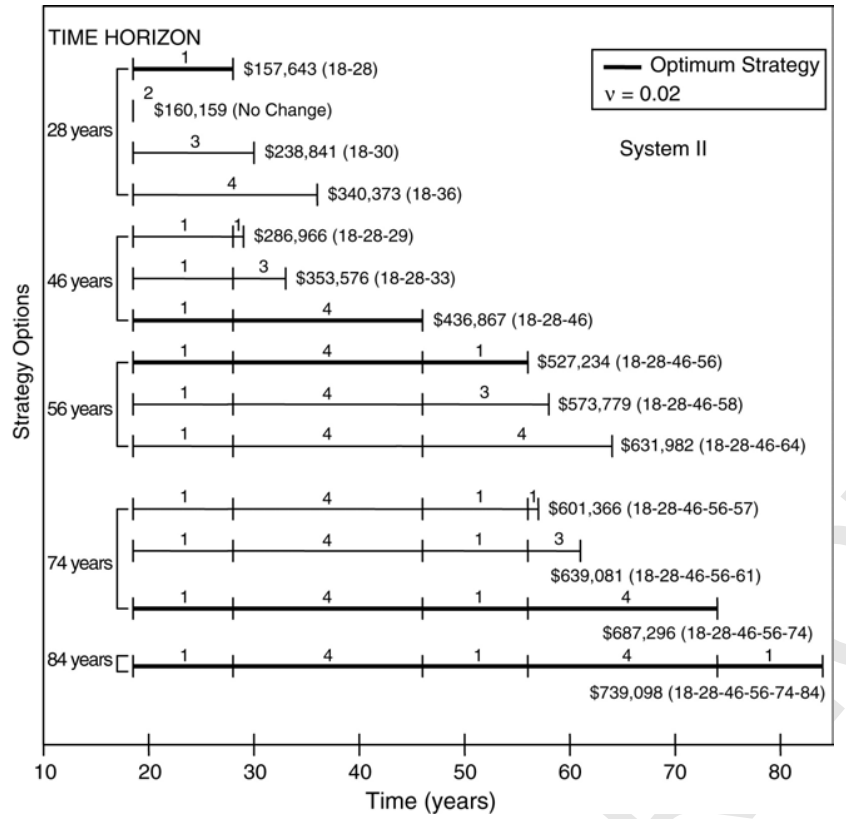

Fig. 15. Optimization of maintenance strategy for bridge system II.

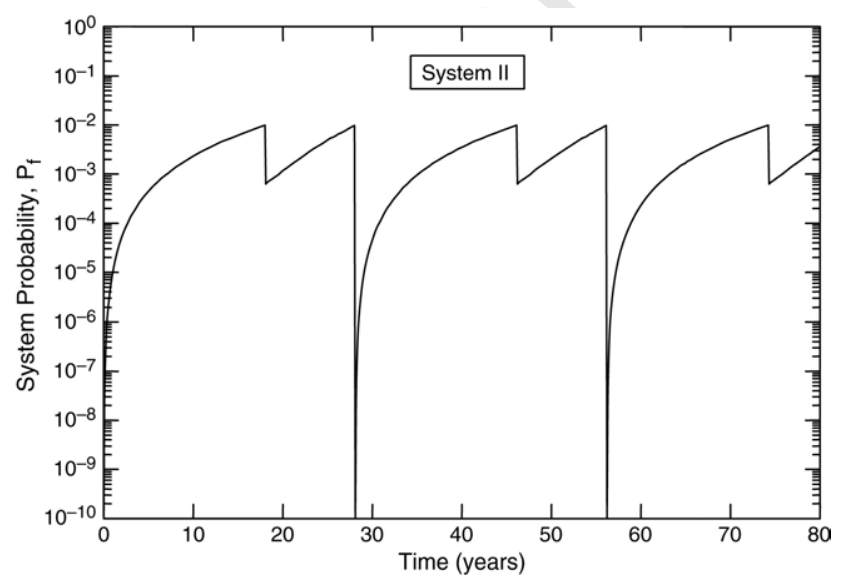

Fig. 16. System probability of occurrence of severity defect 4 under optimum maintenance strategy for bridge system II.

1 discount rates of $0,2,4,6$, and $8 \%$. As expected, the increase 2 in redundancy from system I to III is accompanied by a 3 significant decrease in cost. It is also noted that there is a

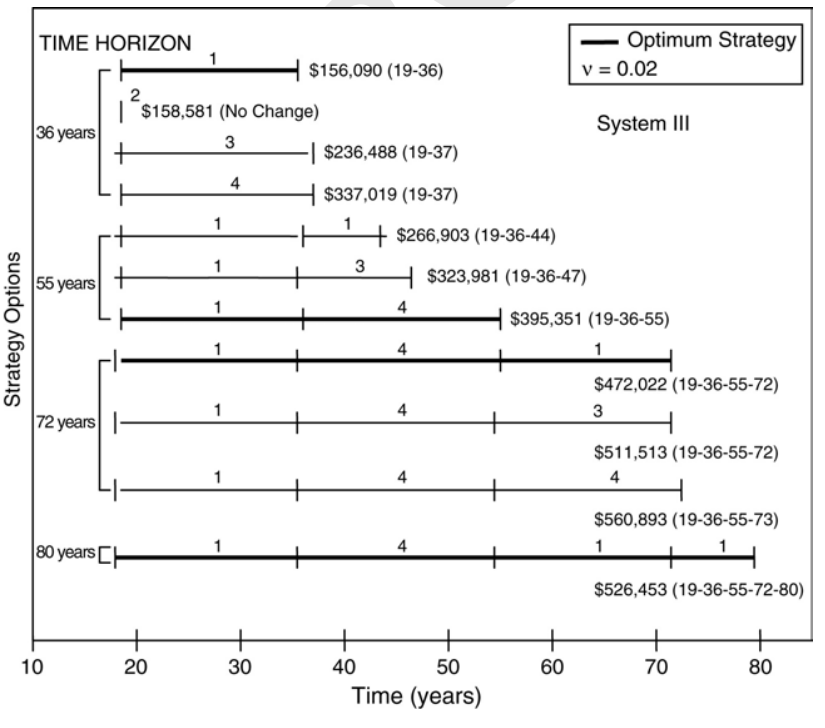

Fig. 17. Optimization of maintenance strategy for bridge system III.

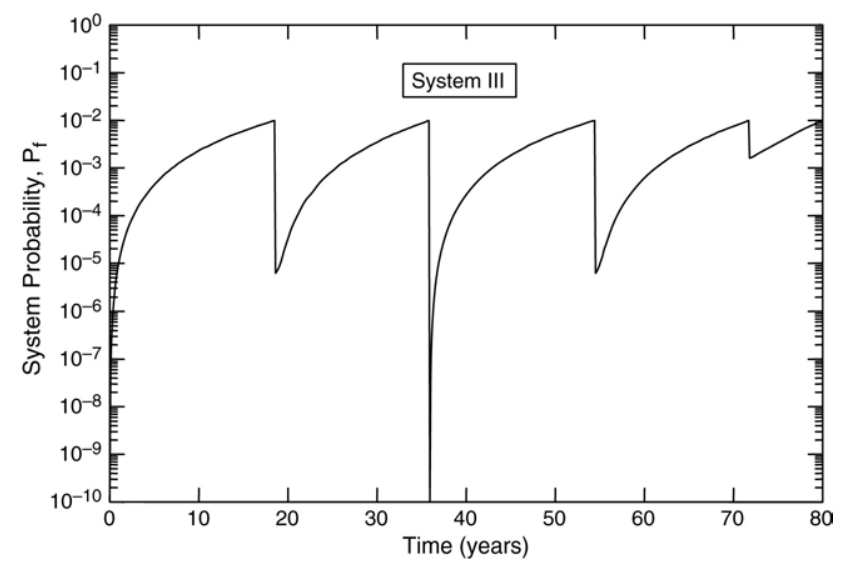

Fig. 18. System probability of occurrence of severity defect 4 under optimum maintenance strategy for bridge system III.

significant change in the present values of optimum lifetime cost due to the discount rate. 


\section{Conclusions}

In this study, a model for predicting the evolution in time of the reliability of deteriorating structures based on lifetime functions is presented. The approach discussed in this paper complements that presented in [25,26]. The effects of proactive, reactive, and essential maintenance on components and systems are studied and models for incorporating these effects in the analysis of deteriorating structures using lifetime functions are discussed.

In this paper, a binary performance indicator is used to decide on the best maintenance strategy for deteriorating structures. This approach is less accurate than the continuous damage model approach, but can be implemented using the information currently available on most structures. Lifetime functions proved to be adequate to model the evolution in time of the performance of deteriorating structures under the effect of maintenance actions. The uncertainty in the lifetime of deteriorating components is captured through Weibull and exponential distributions.

The optimization process based on lifetime functions produces an optimum lifetime maintenance strategy for initial planning purposes. It is therefore important for the optimized plan to be updated based on inspection results [7].

The proposed model is applied to an existing bridge in Denver, Colorado. Several system models, each corresponding to different damage-tolerant policies, are considered for the bridge superstructure and the optimum maintenance strategy for each of these models is computed. The results obtained show significant changes in the optimum strategy and the associated present value of cumulative cost among different system models. Therefore, a correct definition of the system model is crucial in the design, assessment and optimum maintenance planning for deteriorating structures. The present value of cumulative cost of optimum maintenance scenarios, for all system models, is very sensitive to the discount rate.

The use of an analytical model alone is not, however, sufficient to provide an accurate prediction of the future performance of a structure. The optimization of bridge maintenance actions must combine both analytical models and the results obtained from non-destructive tests and visual inspections. In this study, only historical records from visual inspections on similar bridges are used. However, more accurate assessment and prediction of performance will be possible if the results provided by this model are updated using health monitoring information.

\section{Acknowledgements}

The partial financial support of the U.S. National 49 Science Foundation through grants CMS-9912525 and 50 CMS-0217290 is gratefully acknowledged. The support 51 provided by the Colorado Department of Transportation and 52 by the Dutch Ministry of Transportation, Public Works, and
Water Management is also gratefully acknowledged. The opinions and conclusions presented in this paper are those of the writers and do not necessarily reflect the views of the sponsoring agencies.

\section{References}

[1] Bris R, Chatelet E, Yalaoui F. New method to minimize the preventive maintenance cost of series-parallel systems. Reliability Engineering and System Safety 2003;82(3):247-55.

[2] Das PC. Prioritization of bridge maintenance needs. In: Frangopol DM, editor. Case studies in optimal design and maintenance planning of civil infrastructure systems. Reston (VA): ASCE; 1999. p. 26-44.

[3] de Brito J, Branco FA, Thoft-Christensen P, Sorensen JD. An expert system for concrete bridge management. Engineering Structures 1997; 19(7):519-26.

[4] Engelund S, Sørensen JD. A probabilistic model for chloride-ingress and initiation of corrosion in reinforced concrete structures. Structural Safety 1998;20:69-89.

[5] Enright MP, Frangopol DM. Maintenance planning for deteriorating concrete bridges. Journal of Structural Engineering, ASCE 1999; 125(12):1407-14.

[6] Estes AC. A system reliability approach to the lifetime optimization of inspection and repair of highway bridges. Ph.D. thesis. Department Civil, Environmental, and Architectural Engineering, University of Colorado at Boulder; 1997.

[7] Estes AC, Frangopol DM. Repair optimization of highway bridges using system reliability approach. Journal of Structural Engineering, ASCE 1999;125(7):766-75.

[8] Estes AC, Frangopol DM, Foltz SD. Updating reliability of steel miter gates on locks and dams using visual inspection results. Engineering Structures 2004;26(3):319-33.

[9] Frangopol DM, Das PC. Management of bridge stocks based on future reliability and maintenance costs. In: Das PC, Frangopol DM, Nowak AS, editors. Current and future trends in bridge design, construction, and maintenance. London: The Institution of Civil Engineers, Thomas Telford; 1999. p. 45-58.

[10] Frangopol DM, Kong JS, Gharaibeh ES. Reliability-based life-cycle management of highway bridges. Journal of Computing in Civil Engineering, ASCE 2001;15(1):27-34.

[11] Gharaibeh ES, Frangopol DM, Onoufriou T. Reliability-based importance assessment of structural members with applications to complex structures. Computers \& Structures, Pergamon 2002;80(12): 1111-31.

[12] Hawk H, Small EP. The BRIDGIT bridge management system. Structural Engineering International, IABSE 1998;8(4):309-14.

[13] Kececioglu D. Maintainability, availability, \& operational readiness engineering, vol. 1. NJ: Prentice-Hall; 1995.

[14] Kobbacy KAH, Jeon J. Generalized non-stationary preventive maintenance model for deteriorating repairable systems. Quality and Reliability Engineering International 2002;18(5):363-72.

[15] Lam Y, Zhang YL. A geometric-process maintenance model for a deteriorating system under a random environment. IEEE Transactions on Reliability 2003;52(1):83-9.

[16] Lauridsen J, Bjerrum J, Andersen NH, Lassen B. Creating a bridge management system. Structural Engineering International, IABSE 1998;8(3):216-20.

[17] Leemis LM. Reliability, probabilistic models and statistical methods. NJ: Prentice-Hall; 1995.

[18] Maunsell Ltd. Serviceable life of highway structures and their components - final report. Birmingham (UK): Highways Agency; 1999. 
[19] McAllister TP, Ellingwood BR. Evaluation of crack growth in miter gate weldments using stochastic fracture mechanics. Structural Safety 2001;23(4):445-65.

[20] Mori Y, Ellingwood BR. Maintening reliability of concrete structures I: role of inspection and repair. Journal of Structural Engineering, ASCE 1994;120(8):824-45.

[21] Thompson PD, Small EP, Johnson M, Marshall AR. The Pontis bridge management system. Structural Engineering International, IABSE 1998;8(4):303-8.

[22] Tilly GP. Principles of whole life costing. In: Das PC, editor. Safety of bridges. Thomas Telford; 1997. p. 138-44.

[23] Van Noortwijk JM, Klatter HE. The use of lifetime distributions in bridge replacement modeling. In: Casas JR, Frangopol DM, Nowak AS, editors. Bridge maintenance, safety and management. Barcelona: CIMNE; 2002 [8 pages on CD-ROM].
[24] Yang S-I. Predicting lifetime reliability of deteriorating systems with and without maintenance. Ph.D. thesis. Department Civil, Environmental, and Architectural Engineering, University of Colorado at Boulder; 2002.

[25] Yang S-I, Frangopol DM, Neves LC. Service life prediction of structural systems using lifetime functions with emphasis on bridges. Reliability Engineering and System Safety, Elsevier 2004;86(1): 39-51.

[26] Yang S-I, Frangopol DM, Kawakami Y, Neves LC. The use of lifetime functions in the optimization of interventions on existing bridges considering maintenance and failure costs. Reliability Engineering and System Safety, Elsevier 2005 [in press].

[27] Zheng R, Ellingwood BR. Stochastic fatigue crack growth in steel structures subject to random loading. Structural Safety 1998;20(4): 303-23. 